\title{
On the reprocessing of gamma-rays produced by jets
}

\author{
M. Orellana ${ }^{1,2}$, L. J. Pellizza ${ }^{3}$ and G. E. Romero ${ }^{4,1}$ \\ ${ }^{1}$ Facultad de Cs. Astronómicas y Geofísicas - Universidad Nacional de La Plata, Argentina \\ ${ }^{2}$ Departamento de Física y Astronomía, Universidad de Valparaíso, Chile \\ email: morellana@fcaglp.unlp.edu.ar \\ ${ }^{3}$ Instituto de Astronomía y Física del Espacio, UBA - CONICET, Argentina \\ email: pellizza@iafe.uba.ar \\ ${ }^{4}$ Instituto Argentino de Radioastronomía (IAR), CCT La Plata - CONICET, Argentina \\ email: romero@iar.unlp.edu.ar
}

\begin{abstract}
Systems of two very different sizescales are known to produce very high-energy (VHE) radiation in their jets: AGNs and microquasars. The produced VHE photons $\left(E_{\gamma} \sim 1 \mathrm{TeV}\right)$ can be absorbed by the intense environmental soft photon fields, coming from the companion star (in high mass binaries) or from the accreting material (disk+corona in AGNs), as these are the dominant sources at energies around $\sim\left(m_{e} c^{2}\right)^{2} / E_{\gamma}$. Energetic pairs are created by the photonphoton annihilation, and, depending on how efficient are the competing cooling channels, the absorption can lead to a reprocessing by Inverse Compton pair-cascade development. A selfconsistent modeling of these systems as gamma-ray sources should then include, along with the emission and absorption processes, a thorough treatment of the pair cascades. We discuss here on this issue, focusing on our (preliminary) results of numerical simulations devoted to a study case similar to the high-mass microquasar candidate LS 5039.
\end{abstract}

Keywords. radiation mechanisms: nonthermal, gamma rays: theory

\section{Introduction}

Many efforts have been devoted to the calculation of electromagnetic (EM) cascades using both numerical (e.g. Protheroe 1986, Bednarek 1997 and subsequent works, Orellana et al. 2007) or semi-analytical techniques (Aharonian \& Plyasheshnikov 2003). At high energies the angles of emission of secondary electrons and photons are very small, thus the shower develops essentially in the direction of the incident particle (radially away from the source) when one deals with EM cascades in the absence of magnetic fields.

In a more realistic case, the presence of magnetic fields changes the problem dramatically, which become 3D as the electrons are deflected from their original direction $\dagger$. More importantly, the radiative synchrotron channel take out energy of the system and can

$\dagger$ Four different regimes can in principle be distinguished, in similar as Gabici \& Aharonian (2007) pointed out concerning different values of the intergalactic magnetic field: Regime I: $B \ll B_{\text {iso }}$. The EM cascade is not affected at all. The electrons in the cascade do not suffer synchrotron losses, nor are they deflected. Thus, the EM cascade develops along a straight line. Regime II: $B_{\text {iso }} \leqslant B \ll B_{\mathrm{syn}}$. No energy is subtracted to the EM cascade due to synchrotron losses, but low energy electrons are effectively isotropized by the magnetic field. Regime III: $B \geqslant B_{\text {syn }}$. The development of the EM cascade is strongly suppressed since its very first steps due to strong synchrotron losses. Regime IV: $B \gg B_{\mathrm{syn}}$. The magnetic field is strong enough as to maintain a pure synchrotron/curvature cascade, as in the polar regions of pulsars. The inequalities in these items leave a gray area to be explored by detailed calculations. 
take the leading of the energy losses of the charged particles changing the character of the cascade development.

We have devised a scheme for the simulation of electromagnetic cascades in the presence of magnetic fields from an ab initio point of view, and versatile enough as to be applied to many different systems (Pellizza, Orellana \& Romero, 2010). There are three main advantages of our scheme. First, the code can be applied to any $\gamma$-ray production model for which the density, energy and momentum of the primary photons are given, with arbitrary target photon fields and magnetic field configurations. Second, it computes three dimensional cascades that give information on the spectral energy distribution of photons leaving the system, and its dependence with time, position and propagation direction. Finally, it provides a self-consistent treatment of synchrotron emission, and its dependence with position within the physical system and time.

\section{Aplication to LS 5039: preliminary results}

We have computed the cascade produced in the high-mass $\gamma$-ray loud binary LS 5039. Though its nature is not clear, it remains as an excellent microquasar candidate for which the VHE photons produced at the base of the jets triger EM cascades during their propagation (see e.g. Bosch-Ramonet al. 2006, for an emission model, and Cerutti et al. 2010 for alternative cascade treatment). Here we have considered power-law injected photons (photon index 2), through the background stellar radiation field (taken as a black body with $T=3 \times 10^{4} \mathrm{~K}$, and $R_{\star}=10^{12} \mathrm{~cm}$ ), and for simplicity, a constant and isotropic injected luminosity was assumed. Note the low eccentricity and inclination of the system $\left(i \sim 30^{\circ}\right)$ are responsible for a moderate variation in the opacity as a function of the orbital phase.

The ambient (ordered) magnetic field, $B_{0}$, is assumed uniform in a simplifying approach. We take it to be $B_{0}=0.001 \mathrm{G}$, a value that is expected to give EM cascades in the anisotropic regime, which haven't been explored by other authors so far. See e.g. the plot for the cascades domains as estimated by Cerutti et al. (2010), for LS 5039. The pairs are confined by the magnetic field inside the binary system during a certain (nonnegligible) time interval while they radiate by Synchrotron and IC processes. These can lead to modifications in the light curve (delayed from injection) that we are planning to explore in a forthcoming work. The resulting observable spectra, normalized to the total energy injected by primary particles for different orbital phases (phase zero corresponds to the periastron), were obtained and will also be presented elsewhere.

\section{References}

Aharonian, F. A. \& Plyasheshnikov, A. V. 2003, Astropart. Phys. 19, 525

Bednarek, W. 1997, A\&广A 322, 523

Bosch-Ramon, V., Romero, G. E., \& Paredes, J. M. 2006, A\&A 447, 26

Cerutti, B. et al. 2010, AESA, in press [arXiv:1006.2683]

Gabici, S. \& Aharonian, F. A. 2007, Ap\&SS 309, 465

Orellana, M., Bordas, P., Bosch-Ramon, V., Romero, G. E., \& Paredes, J. M. 2007, A\&A 476, 9

Protheroe, R. J. 1986, MNRAS 221, 769

Pellizza, L. J., Orellana, M., \& Romero G. E. 2010, IJMPD, Vol. 19, 671-676 